\title{
High Resolution Actuators for Severe Environments
}

\author{
Christian Belly, Mathieu Bagot, and Frank Claeyssen \\ Cedrat Technologies S.A \\ 15 chemin de Malacher, Inovallée, 38246 Meylan Cedex, France \\ christian.belly@cedrat.com
}

\begin{abstract}
Stepping Piezo Actuators (SPA) are long stroke linear piezoelectric actuators able to perform long stroke (typ. $>10 \mathrm{~mm}$ ) with an important resolution (typ. $<1 \mathrm{~nm}$ ). Use of famous Amplified Piezo Actuator APA ${ }^{\circledR}$ gives the motor interesting characteristics, such as useful deformation mode and speed improvement, compared to standard inertial motors.

The paper proposes experimental results obtained on various size of SPA. Speed aspects and max input current are considered as well as non-magnetic and thermal vacuum compatibilities.
\end{abstract}

Keywords: linear, piezoelectric, motor, actuator, stepping, $\mathrm{APA}^{\circledR}$.

\section{Introduction}

The stroke offered by piezoelectric actuators is often too short to fit the constraints of some specific designs. A classical solution consists in what is called piezoelectric motors. Possibly ultrasonic, inchworm, or inertial, these motors have the particularity to work on friction and so, to have a holding force without any consumption. One of their main drawbacks concerns the lifetime, because of friction wear. The present work is based on Stepping Piezo Actuator (SPA), which is an inertial piezo motor, a promising type in terms of miniaturization but also facing environment constrains, such as high magnetic fields or thermal vacuum.

The concept of inertial piezoelectric motor, introduced in [1], has been used in order to fit extreme precision needed in Tunneling Microscope Scanning [2], compatible with cryogenic environments. A similar concept was proposed by Higuchi in [3], and was adapted to build the Smooth Impact Drive Mechanism [4], used in camera blur reduction mechanism. The aim of this work is to present the new SPA30uXS, miniature inertial piezo motor, taking advantage from mechanical amplification from the APA ${ }^{\circledR}$. The SPA40SM is also presented with evidence of its thermal vacuum compatibility.

\section{Motor Principle}

Stepping Piezo Actuators are inertial stepper motors. They are composed of four main elements to make long stroke and high resolution possible: an actuator, a shaft, a mass and a passive clamp. The actuator is an Amplified Piezoelectric Actuator $\left(\mathrm{APA}^{\circledR}\right)$, widely used in industrial, military and space applications. Its 
reliability comes from the prestress of the ceramic, and the easiness of integration makes this actuator especially relevant. We will see further that the benefits taken from this kind of actuator are wider than these two points.

The principle of such motor is simple and relies on stick-slip effect and dissymmetrical accelerations. Fig. 1 shows the two phases needed to produce one step. First, a slow contraction of the actuator makes the mass moving, without any motion of the shaft, because of clamping friction. Then, a fast actuator expansion gives dynamic forces to mass and shaft and, because of the inertia of the mass, overcomes the friction forces. This moves the shaft into the clamp and one step is completed. By repeating this operation, stroke of several millimeters can be reached. The opposite motion is done by inversing the two sequences. This motion is called "Stepping Mode".

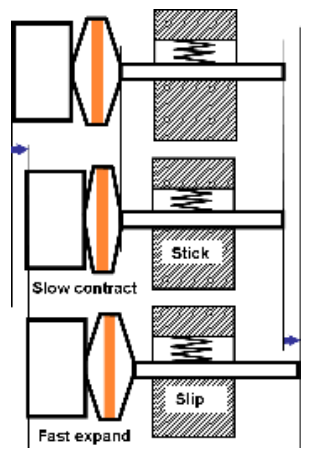

Fig. 1. Concept of Stepping Mode

Another way to use the SPA is called "Deformation Mode". In this case, the tool is attached to the mass and it is the actuator deformation which is used, without dynamic effect. By the way, the precise motion offered by the $\mathrm{APA}^{\circledR}$ allows reaching a high resolution. The Fig. 2 presents an example of motor displacement using combination of stepping mode and deformation mode (with PID controller).

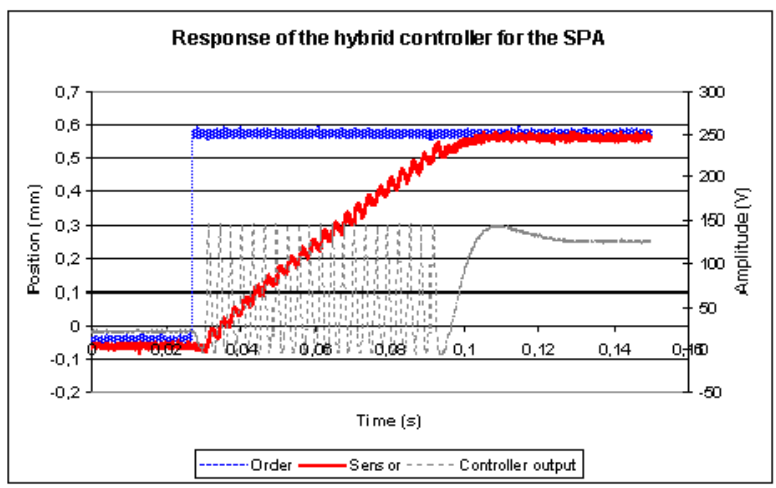

Fig. 2. Combination between stepping and deformation modes 


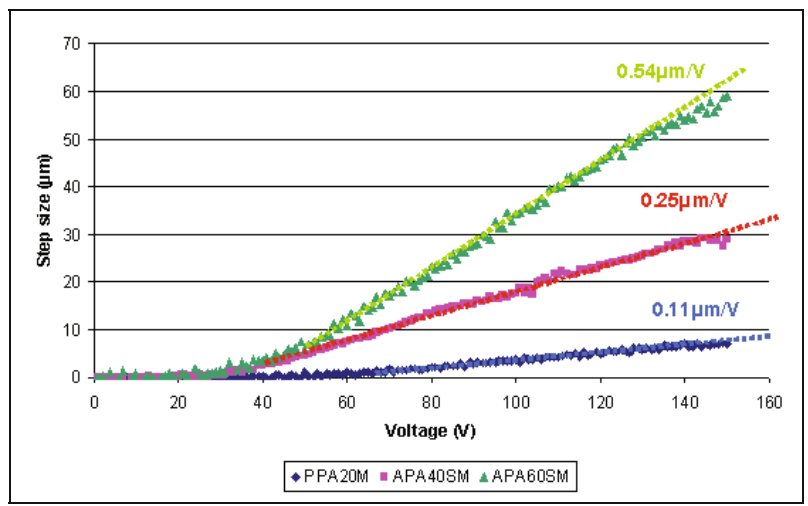

Fig. 3. SPA voltage characteristic

The configuration of the SPA offers qualitative arguments in favor of the amplification and especially in favor of the APA ${ }^{\circledR}$. Firstly, the Deformation Mode is available on a useful stroke $(>30 \mu \mathrm{m})$, making fine adjustment convenient. Secondly, the long stroke of the $\mathrm{APA}^{\circledR}$ makes the design constrains less demanding. The elasticity of the shaft and of the contact can be overcome because of that. Finally, the prestress of the ceramic by the shape of the $\mathrm{APA}^{\circledR}$ gives the motor another reliability argument.

\section{Benefits from Amplification}

SPA is driven using a kind of sawtooth signal. This signal reaches a maximum voltage, which characterizes the amplitude of the deformation of the actuator. This deformation induces the step size and so, it is possible to present the relation between maximum voltage of the input signal and the step size. This curve allows determining the minimum voltage needed to move the motor: the threshold voltage [5]. The second information available is the gain $[\mu \mathrm{m} / \mathrm{V}]$, which determines the capability of the motor to convert voltage into motion.

Experimentation has been led with three different actuators from Cedrat Technologies: PPA20M APA40SM and APA60SM. Each of them presents a different stroke, respectively 20, 40 and $60 \mu \mathrm{m}$. The three characteristics are plot in Fig. 3. It can be seen that, for the three cases, the threshold voltage is quite similar. However, the gain is totally different and it is clear that the bigger is the stroke, the bigger is the gain, making the motor more efficient, in terms of motion and speed.

\section{Miniaturization}

\subsection{Design}

In order to fit extreme volume constrains, a new micro $\mathrm{APA}^{\circledR}$ has been designed: the APA30uXS. Able to reach $40 \mu \mathrm{m}$ of stroke and to produce $3,3 \mathrm{~N}$ force, its stiffness is 
$0,11 \mathrm{~N} / \mu \mathrm{m}$. In the SPA, the $\mathrm{APA}^{\circledR}$ shell, the shaft and the mass are made in one single part, so the reliability of this kinematics group is strongly improved. Moreover, the manufacturing cost and assembling cost are also reduced, by reducing the number of parts to only two. Fig. 4 presents the realized motor. Its weight is less than 2 grams, for a $15 \times 5 \times 9 \mathrm{~mm} 3$ outer volume.

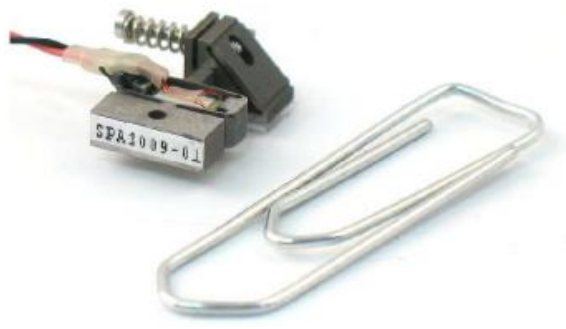

Fig. 4. SPA30uXS

\subsection{Results}

The SPA being built around an $\mathrm{APA}^{\circledR}$, it has been shown that amplification allows reducing current requirement compared to direct actuators [6] in inertial stepper motors. The amplification offered by $\mathrm{APA}^{\circledR}$ is also interesting in terms of step size and speed. In fact, analytical approach of step size, already investigated by Higushi, Breguet and others [7], shows that step size is directly linked to the stroke of the actuator, whatever the input signal is. This point explains that the biggest the stroke is, the bigger can be the step size. Support of Lumped model presented in [5] allows confirming this influence.

This step size conclusion can be extended to the motor speed, taking into consideration that step recurrence (input signal frequency) has to be limited by the natural frequency of the motor. Indeed, if the input signal frequency comes too close or behind the natural frequency, the behavior of the motor may become erratic, creating big speed variation, or even motion direction reversing. Investigations about use of dynamic strain of $\mathrm{APA}^{\circledR}$, especially in SPA, are still in progress [8].

The speed of a SPA30uXS can reach $70 \mathrm{~mm} / \mathrm{s}$, and $0,3 \mathrm{~N}$ actuation force under optimal conditions (shaft preload and signal).

\subsection{Compatibilities}

The SPA30uXS has been designed with totally no-magnetic materials and components in its standard version. Foreseen to be part of biomedical devices or surgical robotics apparatus, the SPA30uXS has been widely tested to check its compliance with Magnetic Resonance Imaging (MRI). Tests have been performed into a 4.7 Tesla magnet, at the Small Animal Facility, INSERM Grenoble, and the three MRI compatibility rules have been proved. The first point is the safety issue: the motor is not attracted by the magnet, so it can be placed into a MRI scanner room and even into the scanner. The second point is the "invisibility issue": the motor does not disturb the 
MR images. Tests have been done putting the motor within the field of view of the scanner and no impact on the Signal / Noise Ratio (SNR) has been observed. Other tests have been done with rat brain observation, reinforcing these results. The third point concerns the insensitivity: the motor is not disturbed by the MRI magnetic fields. This point is harder to observe because of the leak of adapted sensor. So, MR Images have been used to see the motor position into the scanner, using an adapted test bench (see Fig. 5).

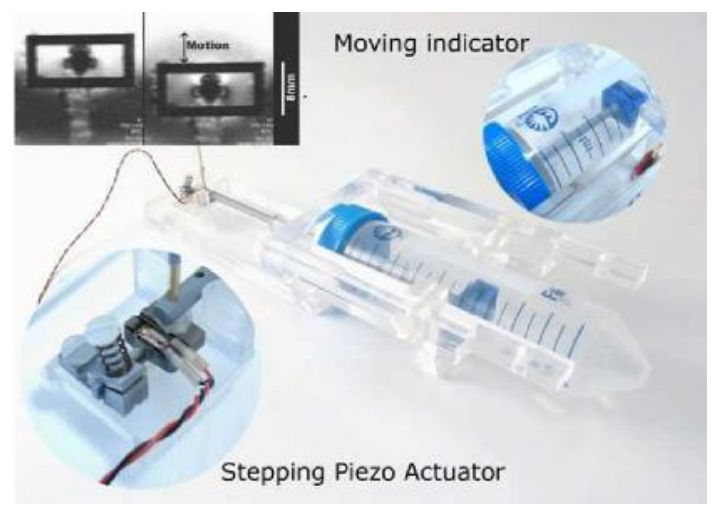

Fig. 5. SPA MRI test bench and results

\section{Force Maximization into Space Environment}

\subsection{Design}

The $\mathrm{APA}^{\circledR}$ concept offers a large range of stroke and force. In case where high force is needed, the use of an APA40SM is preferred. The stroke of such actuator is $52 \mu \mathrm{m}$ and blocked force is 194N. A photograph is this amplified actuator is presented in Fig. 6.

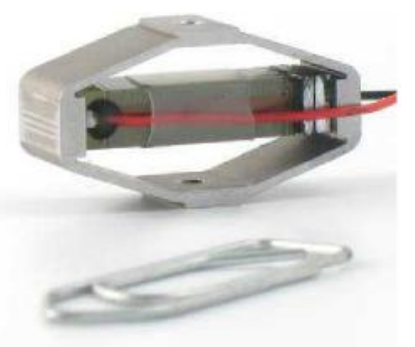

Fig. 6. APA40SM

The clamp system has been designed in order to fit normal and tangential forces and stress. Play recovery system is included and material is chosen to offer good thermo-mechanical behavior. Contact geometry is defined in order to minimize contact pressure between clamp and shaft, allowing lifetime improvement. 
The contact is made between a metal on a friction polymer material. This material couple has been already used in space applications, and showed good results in miniature Stepping Piezo Actuators. The contact interface is longer than the stroke used during functional tests in order to get easy comparison between worn out and virgin contact area.

In order to assess the performance of the motor, a thermal vacuum compatible sensor is needed. For displacement an LVDT sensor was used to give sufficient resolution. This kind of sensor doesn't need any additional bearing. A Sensorex SX20MR005 was used for this test.

The whole motor is thus composed of its four main elements, and is completed by the LVDT sensor, and a loading system, acting on the shaft (Fig. 7). The loading system is composed of a spring which gives the loading characteristics of the motor (see 5.2). The loading spring is calibrated using spring mass resonance frequency measurement.

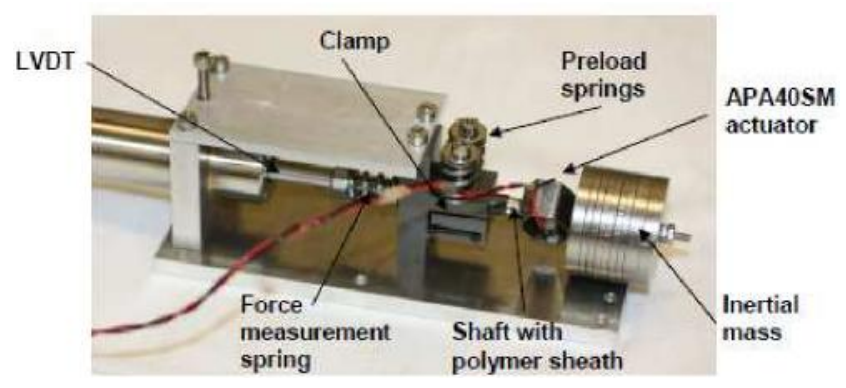

Fig. 7. SPA40SM test bench

\subsection{Results}

According to the motor's actuation profile, the measured motor displacement is naturally dissymmetrical. The positive motion (Fig. 8) plays the role of spring loading. By the way, the motion looks like a capacitance loading curve, loading energy

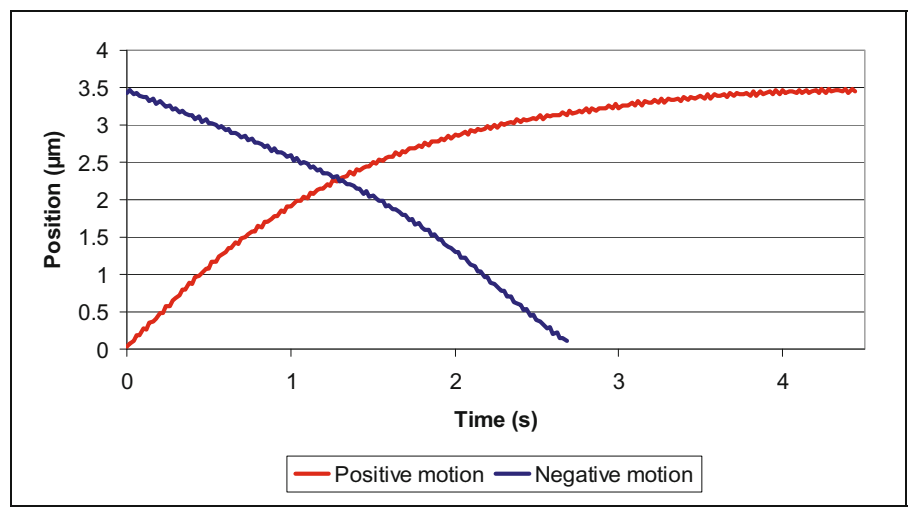

Fig. 8. Positive and negative motion examples 
into spring. In the opposite direction (Fig. 8) the motor speed is not exactly as expected. Indeed, at the beginning of the motion, the motor is pushed by the force measurement spring, so higher speed was expected. As a contrary, lower speed is measured. This shows that motor speed is mainly ruled by the motor itself and not by external forces. At the end of the negative motion, a constant speed is reached.

These two motions examples are used to get the SPA40SM performances for all the thermal vacuum testing, made by ESTL Lab. UK.

\subsection{Compatibilities}

The maximum motor working temperature is limited by the friction material's maximum allowed temperature. For this reason, functional tests were limited to $+60^{\circ} \mathrm{C}$. Tests under vacuum demonstrated that the actuator keeps its performance up to this temperature.

Low temperature tests were performed on the SPA40SM from $0{ }^{\circ} \mathrm{C}$ down to $180^{\circ} \mathrm{C}$, the lowest temperature allowed by the test rig. The results are shown in Fig. 9 . It can be seen that a slight drive force reduction is measured at lower temperatures, but not lower than $75 \%$ of nominal force achieve at ambient temperatures. This reduction seems to be a consequence of the speed reduction of the motor. Indeed, the test bench stops position acquisition if the motion overcomes a certain time. For lower temperatures, this threshold time has been increased, allowing recovering $20 \mathrm{~N}$ drive force measurements. Speed is also affected, but in a coherent way, with a linear speed reduction with temperature. However, after every temperature step, the performance is recovered upon returning to room temperature, showing no continued reduction in performance after periods of exposure to low temperatures under vacuum.

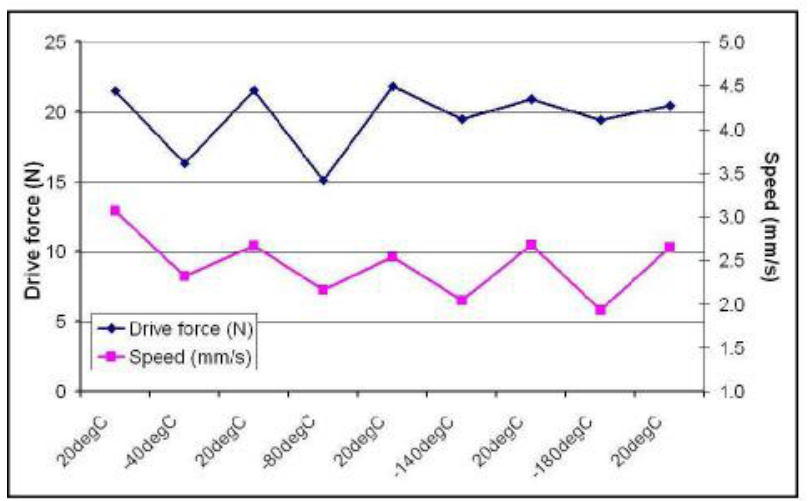

Fig. 9. Low temperature performances

\section{Conclusions}

This paper presents the Stepping Piezo Actuator SPA principle and details the benefits taken from use of Amplified Piezo Actuators APA ${ }^{\circledR}$. The current smallest 
prototype of the Stepping Piezo Actuator SPA30uXS is then presented. The performances reached but also the compatibility of this actuator to fit Magnetic Resonant Imaging MRI constrains are proved. Bigger motor, so called SPA40SM, is also detailed with its vacuum test bench. In this case, high drive forces are obtained from ambient to vacuum and low temperatures, down to $-180^{\circ} \mathrm{C}$. Main performances summary is presented on Table $\mathbf{1}$.

To sum-up, this technology of actuator offers high resolution motion, capable to work in various environments, from MRI to thermal vacuum. Further work will concern complementary guidance to give the motor the correct performances in terms of pitch, yaw and roll, that are not yet considered.

Table 1. SPA motors typical performances

\begin{tabular}{|l|l|l|l|}
\hline & SPA30uXS & SPA35XS & SPA40SM \\
\hline Max Force $(\mathrm{N})$ & 0.3 & 2.0 & 20 \\
\hline Max speed $(\mathrm{mm} / \mathrm{s})$ & 70 & 35 & 5.0 \\
\hline $\begin{array}{l}\text { Deformation stroke } \\
(\mu \mathrm{m})\end{array}$ & 30 & 40 & 50 \\
\hline
\end{tabular}

Acknowledgements. Author would like to acknowledge M. Hervé Mathieu, from INSERM-UJF in Grenoble for his support in MRI-tests. M. Michael Buttery and the ESTL lab, UK, are also acknowledged for testing the SPA40SM into thermal vacuum environment.

\section{References}

1. Pohl, D.W.: Sawtooth nanometer slider: A versatile low voltage piezoelectric translation device. Surface Science 181(1-2), 174-175 (1987)

2. Renner, et al.: A vertical piezoelectric inertial slider. Rev. Sci. Instrum. 61, 965 (1990)

3. Higuchi, T., et al.: Precise Positioner Utilizing Rapid Deformations of a Piezo Electric Element. Journal of the Japan Society of Precision Engineering 54(11), 2107-2112 (in Japanese)

4. Okamoto, Y., et al.: Development of linear actuator using piezoelectric element. Trans. Inst. Electron., Inform. Commun. Eng. A J80-A(10), 1751-1756 (1997)

5. Belly, C., et al.: Small impact drive motors based on amplified piezoelectric actuators. In: SMART 2009 Conference Proc. (2009)

6. Claeyssen, F., et al.: Stepping Piezoelectric Actuators Based on APAs. In: ACTUATOR 2008, Conference Proceedings, pp. 623-626 (2008)

7. Breguet, J.M., et al.: Stick and slip actuators: design, control, performances and applications. In: Micromechatronics and Human Science Proceedings, pp. 89-95 (1998)

8. Claeyssen, F., et al.: Dynamic strain limits of Amplified Piezo Actuators. In: ACTUATOR (2010) 